\title{
IX.
}

\section{Ueber die specifische Wärme des Kaliums.}

\author{
Von \\ V. Regnault.
}

Compt. rend. XXVIII, 325.

Dulong und Petit haben entdeckt, dass zwischen der specifischen Wärme der einfachen Stoffe und ihrem chemischen Aequivalent folgendes merkwürdige Gesetz besteht: Die specifischen Wärmen der einfachen Sloffe verhalten sich umgekehrt wie ihre Atomgewichte. Unter der kleinen Zahl numerischer Resultate, welche diese Physiker als Stütze ihres Gesetzes gaben, fanden sich mehrere, welche diese Relation zu den Atomgewichten, welche sie annehmen, vollständig bestătigten; diese Atomgewichte waren jedoch, wie man später fand, völlig ungenau. Die offenbaren Anomalien welche das Dulong- und Petit'sche Gesetz zeigt, haben lange Zeit die Chemiker abgehalten, sich desselben $\mathrm{zu}$ bedienen, um die Aequivalente der Stoffe festzustellen, welche man nicht ganz vollkommen sicher auf rein chemischem Wege ermitteln konnte.

In meiner Abhandlung über die specifische Wärme der einfachen Stoffe ${ }^{*}$ ) habe ich gezeigt, dass die in jenem Gesetze gefundenen Anomalien herbeigeführt worden sind, durch die schon angenommenen Werthe, welche von Bulong und Petit für mehrere specifische Wärmen gefunden worden waren; aber ich fand auch zugleich, dass das Gesetz nicht den Grad von Genauigkeit habe, welchen man bei ihm annahm, und dass es nur eine Annäherung sei. Diess beruht darauf, dass die Wärmecapacität der Stoffe, wie wir sie durch unsere Versuche bestimmen, einige fremde Elemente einschliesst, welche wir bisher nicht davon zu trennen vermochten; namentlich die latente Dilatationswärme und einen Theil der latenten Schmelzungswărme, welche die Körper nach und nach absorbiren, im Masse wie sie erweichen, oft lange Zeit vor der Temperatur, welche man als ihren Schmelzpunct betrachtet. Die Aenderungen, welche man seit ei-

*) Annales de chimie et de phys. LXXIII. Auszug im Journal $X X V, 129$. 
122 Regnault: Ueberdie specifische Wäme des Kaliums.

niger Zeit in der Bestimmung der chemischen Aequivalente vorgenommen hat, haben im Allgemeinen die Abweichungen vermindert, welche ich in meiner früheren Abhandlung bezeichnet hatte.

Dennoch finden sich unter der grossen Menge einfacher Stoffe, deren specifische Wärme ich bestimmen konnte, drei, welche nicht in das Gesetz hineinpassen, wenn man nicht dic von den Chemikern gewöhnlich angenommenen Aequivalente für sie ändert. Diese sind: Das Uran, das Silber und die Kohle.

Das Uran zeigt eine ganz unerklärliche Abweichung, welche mich bestimmt zu neuen Untersuchungen über dieses Element aufzufordern. Die scbonen Versuche von H. Pélig ot zeigten, dass die Substanz, welche man bisher für metallisches Uran gehalten hatte, ein $0 x y d$ sei, und es gelang demselben das wahre Metall $\mathrm{zu}$ isoliren.

Was das Silber betriff, so würde das Gesetz der specifischen Wärme, für dasselbe ein halb so grosses Aequivalent feststellen, als gewöhnlich von den Chemikern angenommen wirrl. Die Formel des Silberoxydes dürfte dann nicht $\mathrm{AgO}_{g}$, sondern $\mathrm{Ag}_{2} 0$ geschrieben werden; es würde dann analog sein dem Oxydul des Kupfers, $\mathrm{Cu}_{2} \mathrm{O}$, und des Quecksilbers $\mathrm{Hg}_{2} \mathrm{O}$. Dic Gründe, dieses nene Aequivalent anzunehmen, sind vorzugsweise der unbestreitbare Isomorphismus des Schwefelsilbers und des Schwefelkupfers $\left(\mathrm{Ca}_{2} \mathrm{O}\right)$, das es in gewissen, krystallisirten Mineralien in allen Verhältnissen ersetzt; die Analogie des Chlorsilbers mit dem Kupferchlorür $\mathrm{Cu}_{2} \mathrm{Cl}$. Ferner hat man niemals einen Isomorphismus eines Silberoxydsalzes beobachtet, mit einem Salze, dessen 0xyl RO ist. Diese Gründe scheinen mir hinreichend überzeugend, so dass ich nicht zweifle, die Chemiker werden bald das Aequivalent des Silbers annelimen, welches man aus der specifischen Wärme des Metalls ableitet*).

Die Kohle existirt in drei verschiedenen Formen, und zeigt dann auch ganz verschiedene physikalische Eigenschaften. Ich habe gezeigt, dass diesen verschiedenen Zuständen auch sehr abweichende specifische Wärmen entsplechen. Han muss ent-

*) II. Rosc hat bekanntlich diese Gründe, welche für die llalbirung des Atomgewichts des Silbers sprechen, vor längerer Zeit angeführt: Poggend. Annal. XV, 585. XXVIII, 158. G. Rose ibid. p. 433. 
scheiden, welcher Zustand der Kohle es sei, in welchem sie in die chemischen Verbindungen eingehe, und dies ist offenbar der, in welchem ihre specifische Wärme ihrem Aequivalente entspricht.

Es ist natürlich anzunehen, dass dies die Modification sei, in welcher der Kohlenstoff am feinsten vertheilt ist. Die Wärmecapacität nun, welche ich für die, durch Zersetzung der organischen Stoffe durch die Wärme erhaltene Kohle gefunden habe, entspricht dem Aequivalent 150; alșo eine doppelt so grosse $Z$ ahl als sie von den Chemikern bisher fast allgemein angenommen ist. Ich habe früher die Gründe angeführt, welche dazu führen, dieses Aequivalent als das wahrscheinlichste zu betrachten. Unter diesen ist, wie mir scheint, einer ganz entscheidencl. Wir kennen in diesem Augenblick eine grosse Menge von Stoffen, die aus der organischen Natur dargestellt sind, und deren Zusammensetzung mit Sicherheit festgestellt ist. Alle Formeln derselben zeigen die merkwürdige Eigenthümlichkeit, dass die Zahlen der Kohlenstoffaequivalente gerade sind. Diess ist nun ganz natürlich, wenn die Chemiker ein Aequivalent annehmen, welches halb so gross ist, als das wirkliche. Zwei Verbindungen machen allein eine Ausnahme; es sind die, welche dazu gedient haben, um das alte Aequivalent für den Kohlenstoff zu bestimmen: das Kohlenoxydgas und die Kohlensäure; wir haben aber gar keinen Grund, die Formel des Kohlenoxydgases zu schreiben $\mathrm{CO}$ oder $\mathrm{C}_{2} \mathrm{O}_{2}$. Was die Kohlensăure bétriff, so schreiben die Chemiker ihre Formel $\mathrm{CO}_{2}$, weil sie die kohlensauren Salze, welche sich am zahlreichsten darstellen, als neutrale betrachten, wie z. B. der kohlensaure Kalk, der kohlensaure Baryt u. s. w., und als Bicarbonate die alkalischen Carbonate mit der doppelten Menge Kohlensäure. Die Anomalie verschwindet, wenn man diese Jetzten als die neutralen ansieht, wie es bereits einige Chemiker heut zu Tage thun, und dann die sogeuannten neutralen als basische Salze betrachtet.

Zwischen den specifischen Wärmen der zusammengeșetzten Stofle besteht ein ganz ähnliches Geset $z$, wie zwischen denen der einfachen Stoffe, das sich auch in denselben Grenzen bestätigt: Die specifischen Wärmen der susammengesel:ten Stoffe, welche dieselhen chemischen Formeln $\approx$ eigen, verhalten sich unter einander, umgekehrt wie itre Aequivalente. Meine 
Versuche zeigen jedoch, dass, damit dies Gesetz allgemein werde, einige von den Chemikern angenommene Aequivalente geảndert werden müssen.

Die Verbindungen des Silbers, leren Formeln, wenn man die Zusammensetzung des Silberoxyds durch Ag0 bezeichnet, ăhnlich sind den entsprechenden Verbindungen, welche die 0xyde R0 geben, machen constant eine Ausnahme vom Gesetze. Wenn man indessen die Zusammensetzung des Silberoxyds $\mathrm{Ag}_{2} \mathrm{O}$ schreibt, so findet man, dass seine Salze, verglichen mit denen des $\mathrm{Ku}$ pferoxyduls und Quecksilberoxyduls, dem Gesetze der specifischen Wärme vollkommen entsprechen. Die specifischen Wärmen der Verbindungen des Silbers, wie die des metallischen Silbers selbst, führen daher zu dem Schlusse, dass man für das Silber ein Aequivalent aufstellen muss, welches halb so gross ist, als das, welches die Chemiker jetzi annehmen.

Meine Versuche zeigen eine andere Thatsache, welche die Aufmerksamkeit in Anspruch zu nehmen verdient. Man nimmt im Allgemeinen an, dass das Kali und Natron die Formeln KO und $\mathrm{NaO}$ haben müssten, sie zeigen also Formeln, denen des Baryts; Kalkes, der Magnesia, Eisenoxyduls, des Blcis etc. ăhnlich. Bei dieser Annahme indessen machen die Salze der Alkalien und die binären Verbindungen dieser Metalle eine Ausnahme von dem Gesetze der specifischen Wärmen. Wenn man hingegen die Formeln $\mathrm{K}_{2} \mathrm{O}$ und $\mathrm{Na}_{2} \mathrm{O}$ schreibt, so verschwindet die Anomalie. Diese Basen werden dann isomorph mit dem $\mathrm{Cu}_{2} \mathrm{O}$, $\mathrm{Hg}_{2} \mathrm{O}$ und, wenn man das Silberoxyd $\mathrm{Ag}_{2} \mathrm{O}$ schreibt, auch mit diesem. In meiner Abhandlung über die specifische Wärme der zusammengesetzten Verbindungen habe ich die Gründe angegeben, welche die Chemiker veranlassen müssen, diese Veränderung anzunelmen. Die hauptsächlichsten sind folgende: Man kennt kein alkalisches Salz, welches isomorph wäre mit einer Verbindung einer Basis von RO; niemals kann ein alkalisches Oxyd im unbestimmten Verhältniss ein Metalloxyd ho ersetzen. Die Mincralogen nelsmen frcilich eine solche Ersetzung oft all, welche die Aufstellung ihrer Formeln erleichtert; es ist dies indessen eine willkürliche Hypothese, welche lür viele Mineralien, deren Zusammensetzung man genau kennt, bereils verlassen ist. Endlich ist nach Mitscherlich die Form des schwelelsauren 
Silberoxyds dieselbe wie die des wasserfreien, schwefelsauren Natrons.

Um die Bestimmung der Aequivalente der alkalischen Metalle $z u$ vervollständigen war es sehr wünschenswerth, die specifische Wärme des freien Kaliums zu finden. Ich habe diesen Versuch mehrere Male gemacht, bin jedoch dabei auf viele Schwierigkeiten gestossen. Es ist schwer, Kalium im Zustande der Reinheit zu erhalten und so zu behandeln, dass es sich nicht durch Berührung mit Luft verändere; ferner kann man die specifische Wärme nicht über $0^{\circ}$ untersuchen, da sonst das Metall sich erweicht; und dabei eine beträchtliche Menge latenter Schmelzwärme einschliesst. Ich bin dazu gelangt die specifische Wärme sehr genau zu finden, indem ich das Kalium sich in fester Kohlensäure abkühlen liess, und untersuchte, eine wie starke Abkühlung es auf eine bestimmle Menge Steinöl hervorbrachte, welches in einem kleinen Kalorimeter enthalten war. Indem ich denselben Versuch mit einer bekannten Menge Blei machte, konnte ich das Verhältniss der specifischen Wärme dieser Metalle zu einander feststellen. zahlen.

Die derartigen Versuche gaben mir folgende Verhältniss-

$$
\mathbf{5 , 8 3} \quad \mathbf{5 , 7 7} \quad \mathbf{5 , 4 0}
$$

Die beiden ersten Werthe sind zu hoch, da ich es bei den beiden ersten Versuchen nicht vermeiden konnte, dass dem Kalium-Stückchen nicht eine kleine Menge fester Kohlensăure anhing, wodurch eine stärkere Temperatur-Erniedrigung hervorgerufen wurde.

Das Verhältniss der ron den Chemikern angenommenen Aequivalente dieser beiden Metalle ist nun $\frac{1204,5}{490,0}=2,64$, also fast genau die Hälfte von dem Vorhältniss, welches ich in meinen Versuchen gefunden hatte. Wenn man also daher das von mir vorgeschlagene Aequivalent annimmt, so findet man $\frac{1294,5}{245,0}$ $=5,29$, eine Zahl, welche wenig abweicht von der, die ich durch den dritten, genauesten Versuch gefunden hatte.

Die specifische Wärme des Kaliums führt also zu demselben Schlusse, zu welchem bereits die der Kalium-Verbindungen geführt halten: Das Aequivalent des Kaliums muss halbirt, und die Formel der Alkalien $\boldsymbol{h}_{2} O$ geschrieben werden.

Ich suchte nachzuweisen, dass dic Methode, welche ich 
zur Bestimmung der specifischen Wärme des Kaliums angewendet hatte, eine nur sehr geringe Unsicherheit gewährt. $Z u$ dicsem Zwecke nahm icli zwei Körper, deren Wärmeleitung bei Weitem schlechter ist, als die des Kaliums; nämlich Phosphor und Eis. Der Versuch wurde wie beim Kalium gemacht, nur das Steinöl durch Wasser im Calorimeter erhitzt. Indem ich annahm, dass die specifische Wärme des Bleis zwischen - $78^{\circ}$ und $0^{\circ}$ dieselbe bleibe, nämlich 0,0314 , welche ich für dieses $\mathrm{Me}$ tall zwischen $0^{\circ}$ und $160^{\circ}$ gefunden hatte, eine Annahme, welche sich ron der Wahrheit nur sehr wenig entlernen kann, fand ich für die specifische Wärme des Phosphors:

Zwischen $-78^{\circ}$ und $+10^{\circ}$

Zr. Pers ou fand zwischen - 210 und $+70 \quad 0,1740$

Ich fand zwischen $+10^{\circ}$ und $+30^{\circ}$, d. h. unter Unständen, wo der Phosphor bereits merklich erweicht worden war 0,1887

Man sieht, die specifische Wärme des Phosphors, welche ich bei niedriger Temperatur gefunden habe, ist die, weiche man a priori erwarten musste, wenn man annimmt, dass die Wärmecapacität mit der Temperatur sinkt.

Die specifische Wärne des Eisens zwischen $-7^{\circ}$ und $0^{\circ}$

fand ich bei meinem Versuche

Die übereinstimmenden Versuche von Desains und Person gaben 0,504 zwischen $-20^{\circ}$ und $0^{\circ}$; diese Zahl musste höher sein, da die meinige für viel niedrigere Temperaturen bestimmt worden war.

\title{
$\mathbf{X}$. \\ Darstellung der gasförmigen Jod- und Bromwasserstoffisäure.
}

\author{
Von \\ Ch. Irène.
}

(C. R. XXVIII, 478.)

Um Bromwasserstoffgas zu entwickeln, bediene ich mich des krystallisirten, unterphosphorigsauren Kalkes, den man bei der Darstellung des Phosphorwasserstoffgases durch Phosphorcalcium erhilt; ich bringe denselben in einen Kolben oder eine Retorte 